Retraction

\title{
Retraction: Kuo, W.S. et al. Amelioration of Experimentally Induced Arthritis by Reducing Reactive Oxygen Species Production through the Intra-Articular Injection of Water-Soluble Fullerenol. Nanomaterials 2019, 9, 909
}

\author{
Wen-Shuo Kuo ${ }^{1,2,3,+}{ }^{\dagger}$, Chia-Tse Weng ${ }^{4,+}$, Jian-Hua Chen ${ }^{5,6,+}$, Chao-Liang Wu ${ }^{7}$, Ai-Li Shiau ${ }^{8}$, \\ Jeng-Long Hsieh ${ }^{9}$, Edmund Cheung So ${ }^{5,6,10, *}$, Po-Ting Wu ${ }^{11,12,13,14,15, *}$ and Shih-Yao Chen ${ }^{4,9, *}$ \\ 1 School of Chemistry and Materials Science, Nanjing University of Information Science and Technology, \\ Nanjing 210044, China; wskuo88@gmail.com \\ 2 School of Environmental Science and Engineering, Nanjing University of Information Science and \\ Technology, Nanjing 210044, China \\ 3 Center for Micro/Nano Science and Technology, National Cheng Kung University, Tainan 701, Taiwan \\ 4 Department of Internal Medicine, National Cheng Kung University Hospital, College of Medicine, National \\ Cheng Kung University, Tainan 701, Taiwan; ctweng@mail.ncku.edu.tw \\ 5 Department of Anesthesia, An Nan Hospital, China Medical University, Tainan 709, Taiwan; \\ aptx4869jfk@gmail.com \\ 6 Department of Anesthesia, China Medical University, Taichung 404, Taiwan \\ 7 Department of Biochemistry and Molecular Biology, College of Medicine, National Cheng Kung University, \\ Tainan 701, Taiwan; wumolbio@mail.ncku.edu.tw \\ 8 Department of Microbiology and Immunology, College of Medicine, National Cheng Kung University, \\ Tainan 701, Taiwan; alshiau@mail.ncku.edu.tw \\ 9 Department of Nursing, College of Nursing, Chung Hwa University of Medical Technology, Tainan 717, \\ Taiwan; pipi58871053@yahoo.com.tw \\ 10 Graduate Institute of Medical Sciences, Chang Jung Christian, Tainan 711, Taiwan \\ 11 Department of Orthopedics, College of Medicine, National Cheng Kung University, Tainan 701, Taiwan \\ 12 Department of Orthopedics, National Cheng Kung University Hospital Dou-Liou Branch, College of \\ Medicine, National Cheng Kung University, Yunlin 640, Taiwan \\ 13 Department of Orthopedics, National Cheng Kung University Hospital, College of Medicine, National \\ Cheng Kung University, Tainan 701, Taiwan \\ 14 Department of Biomedical Engineering, National Cheng Kung University, Tainan 701, Taiwan \\ 15 Medical Device R \& D Core Laboratory, National Cheng Kung University Hospital, Tainan 701, Taiwan \\ * Correspondence: edmundsotw@gmail.com (E.C.S.); anotherme500@gmail.com (P.-T.W.); \\ z9903038@email.ncku.edu.tw (S.-Y.C.) \\ + The authors contributed equally to this work.
}

Received: 21 February 2020; Accepted: 25 February 2020; Published: 27 February 2020

One of the contributors to the published paper [1] did not provide permission for the data presented to be published and we have therefore taken the decision to retract the paper. We apologize to readers of Nanomaterials. The decision to retract has been taken in agreement with the authors.

MDPI is a member of the Committee on Publication Ethics and takes the responsibility to uphold strict ethical policies and standards very seriously. 


\section{Reference}

1. Kuo, W.S.; Weng, C.T.; Chen, J.H.; Wu, C.L.; Shiau, A.L.; Hsieh, J.L.; So, E.C.; Wu, P.T.; Chen, S.Y. Amelioration of experimentally Induced Arthritis by Reducing Reactive Oxygen Species Production Through the Intra-Articular Injection of Water-Soluble Fullerenol. Nanomaterials 2019, 9, 909. [CrossRef] [PubMed]

(c)

(C) 2020 by the authors. Licensee MDPI, Basel, Switzerland. This article is an open access article distributed under the terms and conditions of the Creative Commons Attribution (CC BY) license (http://creativecommons.org/licenses/by/4.0/). 